\title{
GTP cyclohydrolase I mutations in patients with dystonia responsive to anticholinergic drugs
}

\author{
Paul R Jarman, O Bandmann, C D Marsden, N W Wood
}

\begin{abstract}
Objectives-to investigate the hypothesis that GTP cyclohydrolase I (GCH1) mutations are responsible for the phenotype of highly anticholinergic responsive dystonia in patients with apparent primary torsion dystonia.

Methods-from 107 British patients with clinically diagnosed primary torsion dystonia, seven patients were identified with an excellent response to anticholinergic drugs. All six exons of the GCH1 gene were sequenced in these patients to identify mutations.

Results-three novel GCH1 mutations were identified in two patients. One patient was a compound heterozygote with asymptomatic carrier parents. The clinical phenotype of patients with and without GCH1 mutations was similar. Conclusions-these findings show that a proportion of patients with apparent primary torsion dystonia and a good response to anticholinergic drugs have GCH1 mutations and therefore have a variant of dopa responsive dystonia. The difficulty in distinguishing clinically between patients with and without mutations underscores the importance of considering the diagnosis of a levodopa responsive dystonia in all such patients.
\end{abstract}

(F Neurol Neurosurg Psychiatry 1997;63:304-308)

Keywords: dopa responsive dystonia; anticholinergic drugs; GTP cyclohydrolase

Anticholinergic drug therapy is widely recognised to be of benefit in some patients with primary torsion dystonia. Benzhexol hydrochloride (Artane) taken at high dosage (up to 120 mg daily) has been shown to result in significant improvement of symptoms in some patients with dystonia but the response is variable and often poorly sustained. ${ }^{1}$ However, there have been reports of dramatic and sustained improvement in patients with torsion dystonia treated with anticholinergic drugs at low dosage..$^{2-6}$

In 1952 Corner reported two siblings with severe generalised dystonia both of whom had shown a remarkable response to benzhexol therapy at a dose of 7 to $8 \mathrm{mg}$ daily. ${ }^{2}$ Dystonia in a paternal uncle indicating autosomal dominant inheritance with reduced penetrance, and the clinical description, including prominent diurnal variation in symptoms, suggest that these patients may have had dopa responsive dystonia. Subsequently Nygaard et al provided an update on one of the siblings, whose response to low dose benzhexol had been maintained for 30 years, and described another patient with a similar response. Both had been switched to levodopa with good results supporting the belief that these patients had dopa responsive dystonia. ${ }^{6}$ Several other cases of generalised dystonia with a pronounced response to low dose anticholinergic therapy have been described and have led to suggestions that dopa responsive dystonia underlies many such cases.

Segawa et $a l^{7}{ }^{8}$ provided the first detailed clinical description of dopa responsive dystonia and described the distinctive clinical phenotype and the dramatic therapeutic response to low doses of levodopa. Patients typically present in childhood with dystonia involving the lower limb which progresses to become generalised unless treated. Diurnal variation of symptoms with improvement of dystonia after sleep is a characteristic feature of dopa responsive dystonia. It is now recognised that the dopa responsive dystonia phenotype may encompass atypical presentations including parkinsonism, spastic paraplegia, and a presentation mimicking athetoid cerebral palsy. ${ }^{9}{ }^{10}$ Most cases are inherited as an autosomal dominant trait with reduced penetrance and females outnumber males by about four to one. Linkage between the dopa responsive dystonia locus and markers on chromosome $14 \mathrm{q}$ led to the identification of mutations within the GTP cyclohydrolase I (GCH1) gene in several cases of typical dopa responsive dystonia. ${ }^{11}{ }^{12} \mathrm{GCH} 1$ catalyses the initial and rate limiting step of tetrahydrobiopterin synthesis. Tetrahydrobiopterin is an essential cofactor for tyrosine hydroxylase, the rate limiting enzyme in the dopamine synthesis pathway.

To investigate the possibility that $\mathrm{GCH} 1$ mutations are responsible for the phenotype of anticholinergic responsive dystonia, we identified seven index patients previously diagnosed as having primary torsion dystonia but responding well to benzhexol. Here we describe the results of mutation analysis of the $\mathrm{GCH} 1$ gene in these patients.

\section{Patients and methods}

PATIENTS

From a survey of 107 British patients ${ }^{13}$ with generalised, multifocal, and segmental dystonia fulfilling the agreed criteria for primary torsion dystonia $^{14}$ we identified 84 patients whose response to treatment with anticholinergic drugs was known. Of these, seven patients were 
Table 1 Summary of GCH1 mutations identified

\begin{tabular}{llllll}
\hline Patient & Exon & Codon change & $\begin{array}{l}\text { Protein } \\
\text { alteration }\end{array}$ & $\begin{array}{l}\text { Restriction site } \\
\text { created/abolished }\end{array}$ & $\begin{array}{l}\text { Control chromosome } \\
\text { mutations }\end{array}$ \\
\hline 2 & 6 & AAA $\rightarrow$ TAA & Lys224Stop & Alu-I+ & $0 / 210$ \\
2 & 1 & CCC $\rightarrow$ CTC & Pro23Leu & Taq-I+ & $1 / 210$ \\
7 & 1 & GAT $\rightarrow$ AAT & Asp115Asn & Dde-I- & $0 / 210$ \\
\hline
\end{tabular}

(+/- indicates creation or abolition of restriction site respectively).

$\star$ Disrupts -1 position of exon 1-intron 1 splice donor site.

studied, all of whom had obtained an excellent response to benzhexol at doses ranging from 15-120 mg daily such that dystonia was completely or virtually abolished. Two patients (4 and 5) had received a trial of levodopa treatment with no improvement in symptoms but were included in the study because it was not known if the dose given was sufficient to exclude a clinical response.

\section{DNA SEQUENCING}

DNA was extracted from peripheral blood lymphocytes using standard techniques. All six exons of the GCH1 gene were amplified separately using the polymerase chain reaction (PCR). PCR primers and conditions were as described previously. ${ }^{10}$

Purified products from the PCR reactions were sequenced using an automatic DNA sequencer (Applied Biosystems 373A). For exon 1, sequencing was performed with fluorescently labelled dye terminators using a T7 sequencing kit (Applied Biosystems). A Taq dye deoxy terminator cycle sequencing kit (Applied Biosystems) was used for sequencing of exons 2-6. Sequencing primers were as described previously. ${ }^{10}$ Both sense and antisense strands were sequenced for each sample. The Sequence Editor version 1.0.3 programme (Applied Biosystems) was used for detection of sequence variations from controls and the published sequence.

RESTRICTION SITE ANALYSIS

The presence of mutations was verified by identification of gain or loss of restriction enzyme recognition sites created by point mutations. Restriction site analysis was then used to screen 210 ethnically matched control chromosomes for the presence of these mutations. Exons were amplified by PCR and the product digested with enzyme according to manufacturer's instructions. Digestion prod-

PATIENT 2

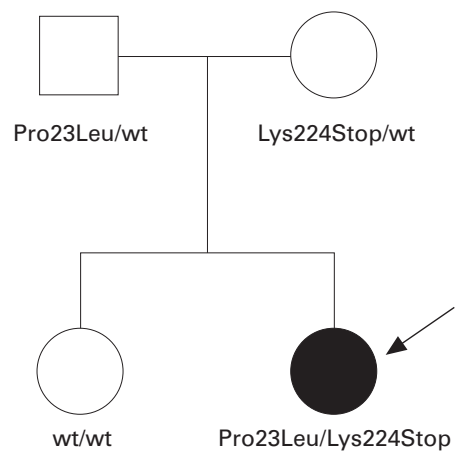

ucts were separated by electrophoresis on a $3.2 \%$ agarose gel containing ethidium bromide.

\section{Results}

MOLECULAR GENETIC ANALYSIS

Three heterozygote mutations of the GCH1 gene were identified in two patients (table 1). All mutations resulted in changes in highly conserved regions of the gene.

In patient 2, a transversion in exon 6 resulted in a premature stop codon at position 224. A transition causing a proline to leucine amino acid change at codon 23 in exon 1 was also identified in this patient. These mutations create new Alu-I and Taq-I cleavage sites respectively. Restriction site analysis disclosed the codon 23 mutation to be present in one of 210 control chromosomes screened (in a male). The other mutation was not found in controls. The parental genotypes for patient 2 were determined by restriction site analysis, showing the Lys224Stop mutation to be present in the mother and the Pro23Leu variant in the father (figure). Both parents were asymptomatic and normal on examination (in the afternoon) by two neurologists.

A missense mutation in patient 7 resulted in an aspartic acid to asparagine amino acid change at residue 115 caused by a substitution in the last nucleotide of exon 1 . The mutation would also be predicted to alter the splice site sequence at the exon 1 -intron 1 boundary. This mutation abolishes a Dde-I restriction site and was not found in any controls. This patient was a sporadic case with no family history of dystonia. However, the patient's father and brother also had the mutation but manifested no features of the disease (figure). An unaffected sister did not have the mutation.

No sequence variants were identified in the remaining five patients.

\section{CLINICAL PHENOTYPE}

Table 2 summarises the clinical characteristics of the patients studied. Dystonia started in the lower limb in all seven patients. The average age at onset was 8.7 years (range 5 to 20 years).

Dystonia progressed to become generalised in five patients and was segmental and multifocal in one patient each. The clinical features in both patients with GCH1 mutations were

Family trees of patients 2 and 7 showing GCH 1 genotypes. Index patients indicated by arrows. Wt $=$ wild type allele. 
Table 2 Summary of patient clinical features and mutations identifed

\begin{tabular}{llllllllll}
\hline Patient & $\begin{array}{l}\text { Distribution of } \\
\text { dystonia }\end{array}$ & Sex & $\begin{array}{l}\text { Age at } \\
\text { onset }(y)\end{array}$ & Age $(y)$ & $\begin{array}{l}\text { Site of } \\
\text { onset }\end{array}$ & $\begin{array}{l}\text { Family } \\
\text { history }\end{array}$ & $\begin{array}{l}\text { Benzhexol } \\
\text { daily dose }\end{array}$ & $\begin{array}{l}\text { Diurnal } \\
\text { variation }\end{array}$ & $\begin{array}{l}\text { Mutation } \\
\text { identified }\end{array}$ \\
\hline 1 & Generalised & F & 8 & 25 & Foot & Yes & $90 \mathrm{mg}$ & $\begin{array}{l}\text { No } \\
\text { Yes }\end{array}$ & $\begin{array}{l}\text { None } \\
\text { Pro23Lu, }\end{array}$ \\
2 & Generalised & F & 5 & 20 & Foot & No & $20 \mathrm{mg}$ & Lys224Stop \\
& Generalised & M & 5 & 25 & Leg & Yes & $35 \mathrm{mg}$ & No & None \\
3 & Multifocal & M & 7 & 26 & Leg & No & $120 \mathrm{mg}$ & No & None \\
5 & Segmental & M & 9 & 19 & Foot & Yes & $30 \mathrm{mg}$ & No & None \\
6 & Generalised & F & 20 & 38 & Leg & Yes & $60 \mathrm{mg}$ & No & None \\
7 & Generalised & F & 7 & 20 & Foot & No & $15 \mathrm{mg}$ & No & Asp115Asn \\
\hline
\end{tabular}

broadly similar to the group as a whole, with onset of dystonia in the lower limb in childhood, progressing to more generalised dystonia before treatment with benzhexol. The mean daily dose of benzhexol required to abolish symptoms was lower in patients harbouring GCH1 mutations (15 mg and $20 \mathrm{mg}$ ), when compared with patients without mutations (range 30-120 mg, mean $67 \mathrm{mg}$ ).

Patient 2 began to toe walk at the age of five. Within three years dystonia had spread to involve all limbs, the trunk, and the muscles of the neck. Dystonia was slightly less severe in the mornings. By the age of eight when treatment with benzhexol was started, she was still able to walk but required the use of a wheelchair for long distances. Within one week of starting treatment with benzhexol, dystonia was virtually abolished. At a dose of $20 \mathrm{mg}$ daily there was no evidence of dystonia on clinical examination.

Patient 7 developed in-turning of the right foot at the age of seven. Over the subsequent two years she developed dystonia affecting both legs, the trunk, and the left hand and arm. There was no diurnal variation in symptoms. The response to benzhexol was apparent within four days and continued to develop over six weeks with almost complete abolition of dystonia, maintained at a dose of $15 \mathrm{mg}$ daily. The patient has subsequently been switched from benzhexol to levodopa (Sinemet-plus three daily). On this regime she is asymptomatic with no dystonia on clinical examination.

Interestingly, both patients with GCH1 mutations were apparently sporadic cases, by contrast with four of the remaining five index patients, in whom the family history was compatible with autosomal dominant inheritance. The mother of patient 5 and the mother, brother, and daughter of patient 6 had primary torsion dystonia. In two patients (1 and 3), family members were asymptomatic but on examination were classified as affected on the basis of writer's cramp, dystonic posturing of the outstretched hands, and pronounced postural tremor. No patients had Ashkenazi Jewish ancestry.

\section{Discussion}

Primary torsion dystonia is an autosomal dominant disorder with reduced (30-40\%) penetrance and variable expression. ${ }^{13}$ Genetic linkage analysis has identified one locus, DYT 1 , on the distal long arm of chromosome 9 in one large non-Jewish and several Jewish families. ${ }^{15}{ }^{16}$ However, a significant proportion of non-Jewish families with primary torsion dystonia are not linked to the DYT 1 locus, indicating the existence of other genetic loci for primary torsion dystonia. ${ }^{17}$

We identified three novel mutations in the GCH1 gene in two out of seven patients diagnosed with primary torsion dystonia, all of whom showed an excellent response to anticholinergic drug therapy. These findings indicate that these two patients have a variant of dopa responsive dystonia.

The mutations in patient 2 are of particular interest. This patient is a compound heterozygote with Lys224Stop inherited from her mother and Pro23Leu inherited from her father. The non-sense mutation found in exon 6 creating a stop codon would be predicted to result in premature truncation of the $\mathrm{GCH} 1$ protein with loss of the $C$ terminal $10 \%$ of the protein. It is interesting to note that a missense mutation causing a conservative amino acid change (a change to a biochemically similar amino acid) has been identified in the same codon in another British family with an unusual presentation of dopa responsive dystonia resembling athetoid cerebral palsy. ${ }^{10}$ The Pro23Leu mutation in the paternal GCH1 allele was found in one of 210 control chromosomes examined. There are two possible explanations for this finding: either Pro23Leu could represent a rare variant in the population, or alternatively, the mutation in combination with a second mutation in the maternal allele may be sufficient to reduce GCH1 activity below a critical threshold whereas neither alone is sufficient to cause disease in either of the carrier parents. Pro23Leu results in a nonconservative amino acid change in a highly conserved region of the gene which suggests that it is a pathogenic rather than neutral mutation. All GCH1 mutations so far identified have been private mutations, so our finding of two apparently unrelated persons (patient 2 and one control) with the same mutation is unusual but may reflect the fact that many previous studies have not screened controls for the presence of mutations. Furthermore, the control subject in whom the mutation was found was male and he may therefore be a nonmanifesting carrier of dopa responsive dystonia similar to the father of patient 2 who is the transmitting carrier of the mutation Very reduced penetrance is the rule rather than the exception in this condition and Ichinose et al have shown that asymptomatic male gene carriers have higher enzyme activities than affected females with the same mutation. ${ }^{12}$ Homozygote missense mutations of $\mathrm{GCH} 1$ result in undetectable enzyme activity and 
patients typically present in infancy with hyperphenylalaninaemia, severely retarded development, abnormal muscle tone, and convulsions. ${ }^{18}$

The parents of patient 2 are both asymptomatic gene carriers with no manifestations of dopa responsive dystonia. This is the first report of compound heterozygote mutations of $\mathrm{GCH} 1$ in a patient with dystonia, and therefore raises the possibility that dystonia due to GCH1 deficiency may be inherited as an autosomal recessive trait in a minority of cases. The patient continues to maintain an excellent response to benzhexol with complete abolition of dystonia, and has not switched to levodopa at her own request.

Two male relatives of patient 7 (Asp115Asn) are non-manifesting carriers. Interestingly, this mutation may disrupt a splice donor site as well as changing the amino acid at residue 23. G->A mutations at the -1 position of the exon-intron boundary have been shown to disrupt RNA transcription in some other diseases. ${ }^{19-22}$ Splice site mutations within introns have been previously described in dopa responsive dystonia and RNA studies are underway in this patient. ${ }^{23}{ }^{24}$ Recently levodopa was substituted for benzhexol in this patient with a good response.

Five of our patients had no identifiable mutation within the coding region of the GCH1 gene. This does not exclude the possibility that these patients have a variant of dopa responsive dystonia as up to half of the cases of clinically definite dopa responsive dystonia have no identified GCH1 mutation. ${ }^{25}$ Mutations outside the coding region of $\mathrm{GCH} 1$ or in a regulatory gene, or gene coding for another enzyme of the tetrahydrobiopterin synthesis pathway could explain such cases. The absence of diurnal variation of symptoms and the higher dose of benzhexol required to produce a response in patients without identified mutations support the supposition that these patients do not have dopa responsive dystonia but rather a variant of primary torsion dystonia. It has been suggested that patients with primary torsion dystonia respond more slowly to anticholinergic drugs than patients with dopa responsive dystonia as well as showing a generally poorer response and needing higher doses. ${ }^{6}$ The difficulty in distinguishing between primary torsion dystonia and dopa responsive dystonia on the basis of clinical features alone makes a therapeutic trial of levodopa mandatory.

Not all patients with clinically definite dopa responsive dystonia respond to anticholinergic drugs. ${ }^{5}$ Nygaard et al found that 17 patients with dopa responsive dystonia had previously tried an anticholinergic drug with varying degrees of success. ${ }^{6}$ Overall, most patients with dopa responsive dystonia derived some benefit from anticholinergic drugs, but in most, levodopa therapy was judged superior in terms of both efficacy and adverse effects. Anticholinergic therapy is therefore not a substitute for levodopa in dopa responsive dystonia.

In summary, we have identified three novel GCH1 mutations in two patients with anti- cholinergic responsive dystonia, suggesting that a variant of dopa responsive dystonia underlies dystonia in a minority of patients with apparent primary torsion dystonia. Our findings highlight the difficulty of distinguishing clinically between primary torsion dystonia and dopa responsive dystonia. A therapeutic trial of levodopa is therefore essential in all cases of dystonia with onset in childhood or early adult life even in the absence of any diurnal fluctuation of symptoms or family history. A favourable response to anticholinergic drugs in patients with what seems to be primary torsion dystonia should alert clinicians to the possibility that such patients have a levodopa responsive dystonia.

PRJ is supported by a Wellcome Research Training Fellowship. We thank all patients and referring clinicians.

1 Fahn S. High dosage anticholinergic therapy in dystonia. Neurology 1983;33:1255-61.

2 Corner BD. Dystonia musculorum deformans in siblings. Treated with Artane (trihexyphenidyl). Proc $R$ Soc Med 1952;45:451-2

3 Burns CLC. The treatment of torsion spasm in children with trihexyphenidyl (Artane). The Medical Press 1959;241: $148-9$.

4 Mucklow ES, Metz HT. Remarkable response to benzhexol hydrochloride (Artane) in children with an unusual progressive striatal syndrome. Dev Med Child Neurol 1964; 6:598-605.

5 Harwood G, Hierons R, Fletcher NA, Marsden CD. Lessons from a remarkable family with dope-responsive dystonia. F Neurol Neurosurg Psychiatry 1994;57:460-3.

6 Nygaard TG, Marsden CD, Fahn S. Dopa-responsive dystonia: long-term treatment response and prognosis. dystonia: long-term treatmen

7 Segawa M, Ohmi K, Itoh S, Aoyama M, Hayakawa H. Childhood basal ganglia disease with remarkable response to L-dopa, hereditary basal ganglia disease with marked diurnal fluctuation. Shinryo (Tokyo) 1971;24:667-72.

8 Segawa M, Hosaka A, Miyagawa F, Nomura Y, Imai H. Hereditary progressive dystonia with marked diurnal variation. In: Eldridge R, Fahn S, eds. Advances in neurology. New York: Raven Press, 1976:215-33.

9 Nygaard TG, Marsden CD, Duvoisin RC. Dopa-responsive dystonia. Adv Neurol 1988;50:377-84.

10 Bandmann O, Nygaard TG, Surtees R, Marsden CD, Wood NW, Harding AK. Dopa responsive dystonia in British patients: new mutations of the GTP-cyclohydrolase I gene and evidence for genetic heterogeneity. Hum Mol Genet 1996;5:403-6.

11 Nygaard TG, Wilhelmsen KC, Risch NJ, et al. Linkage mapping of dopa-responsive dystonia (DRD) to chromomapping of dopa-responsive dystonia

12 Ichinose H, Ohye T, Takahashi E, et al. Hereditary progressive dystonia with marked diurnal fluctuation caused by mutations in the GTP cyclohydrolase I gene. Nat Genet 994;8:236-42

13 Fletcher NA, Harding AK, Marsden CD. A genetic study of idiopathic torsion dystonia in the United Kingdom. Brain 1990;113:379-95

4 Fahn S. Marsden CD, Caln DB. Classification and investigation of dystonia. In: Marsden CD, Fahn S, eds Movement disorders 2. London: Butterworths, 1987:332-58.

15 Ozelius L, Kramer PL, Moskowitz CB, et al. Human gene for torsion dystonia located on chromosome 9q32-q34. Neuron 1989;2:1427-34.

16 Kramer PL, de Leon D, Ozelius L, Risch N. Bressman SB, Brin MF. Dystonia gene in Ashkenazi Jewish population is located on chromosome 9q32-34. Ann Neurol 1990;27: 114-20.

17 Warner TT, Fletcher NA, Davis MB, et al. Linkage analysis in British and French families with idiopathic torsion dystonia. Brain 1993;116:739-44.

18 Ichinose $\mathrm{H}$, Ohye T, Matsuda Y, et al. Characterisation of mouse and human GTP cyclohydrolase I genes. Mutations in patients with GTP cyclohydrolase I deficiency. F Biol Chem 1995;270:10062-71

19 Gardella R, Belletti L, Zoppi N, Marini D, Barlati S, Colombi M. Identification of two splicing mutations in the collagen type VII gene (COL7A1) of a patient affected by the localista variant of recessive dystrophic epidermolysis bullosa. Am F Hum Genet 1996;59:292-300.

20 Stockler S, Isbrandt D, Hanefeld F, Schmidt B, von Figura K. Guanidinoacetate methyltransferase deficiency: the first inborn error of creatine metabolism in man. Am $\mathcal{F} \mathrm{Hum}$ Genet 1996;58:914-22.

21 Akli S, Chelly J, Mezard C, Gandy S, Kahn A, Poenaru L. A ' $G$ ' to 'A' mutation at position -1 of a 5 ' splice site in a late infantile form of Tay-Sachs disease. $\mathcal{F}$ Biol Chem 1990;265: infantile for 7324 .

22 Weil D, D'Alessio M, Ramirez F, et al. Temperaturedependent expression of a collagen splicing defect in the 
fibroblasts of a patient with Ehers-Danlos syndrome type VII. 7 Biol Chem 1989;264:16804-9.

23 Hirano M, Tamaru Y, Nagai Y, Ito H, Imai T, Ueno S. Exon skipping caused by a base substitution at a splice site in the GTP cyclohydrolase I gene in a Japanese family with hereditary progressive dystonia dopa responsive dystonia Biochem Biophys Res Commun 1995;213:645-51.
24 Furukawa Y, Shimadzu M, Rajput AH, et al. GTPcyclohydrolase I gene mutations in hereditary progressive and dopa-responsive dystonia. Ann Neurol 1996;39:609-

25 Ichinose $\mathrm{H}$, Ohye $\mathrm{T}$, Segawa M, et al. GTP cyclohydrolase I gene in hereditary progressive dystonia with marked diurnal fluctuation. Neurosci Lett 1995;196:5-8.

\section{HISTORICAL NOTES}

\section{Binswanger's "encephalitis subcorticalis chronica progressiva"}

Otto Binswanger (1852-1929), Professor of Psychiatry in Jena, in 1894 described eight patients with a progressive dementia punctuated by apoplectiform focal attacks whose brains showed diffuse or patchy but selective white matter atrophy, most marked in the temporooccipital regions with ventricular dilatation. He called it "encephalitis subcorticalis chronica progressiva". His original description is often misquoted but Förstl's translation now makes Binswanger's work accessible ${ }^{1}$ :

\footnotetext{
CLINICAL CRITERIA ( $\mathrm{p} 1184$ )

"The disease begins at the onset of senility (early in the fifties) or in advanced old age (early in the sixties);

"slow impairment of intellectual capabilities manifesting primarily by the progressive impairment and ultimate loss of the association between cortical sensory and motor areas;

"most frequently observed are aphasic disturbances (as in the present case), hemiamblyopia or hemianopia, hemiparesis with loss of the sense of pressure, position or touch; these circumscribed deficits are of a stable character during the fully developed disease and they are combined with the slow and relentless deterioration of intellectual performances; ( . . .until) the patients resemble decerebrate laboratory animals.'

NEUROPATHOLOGICAL CRITERIA (p 1137)

"We find a pronounced atrophy of the hemispheric white matter, either restricted to one or more gyri in one brain area or of several hemispheric regions affected with variable severity;

"these changes are most clearly found in the area of the occipital and temporal lobes, so that temporal and occipital horns are widened into bag-like cavities, while the anterior portion of the lateral ventricle shows relatively little enlargement and the frontal white matter is almost unaffected by the disease process. ... The cortex does not show any remarkable macroscopic change apart from a slight narrowing. Invariably, these cases show severe atheroma of the cerebral arteries. . it is very likely that the subcortical loss of fibres is caused by a deficiency of the blood supply resulting from arteriosclerosis."

Binswanger did not describe the microscopic pathology, but Alzheimer did give a more detailed account of
}

the histology, and named the condition "Binswanger's disease." Alzheimer reported:

"One can show in the white matter, the presence of more or less numerous foci which produce wide areas of secondary degeneration... Usually the foci are also to be found in the internal capsule, the lenticular nucleus, the thalamus, and particularly in the pons in the region of the pyramidal tract... caused by a particularly severe arteriosclerosis of the long vessels deep in the white matter with intense atrophy of the white matter." ${ }^{2}$

The distinction from senile dementia, general paralysis of the insane, and mixed Alzheimer's disease with multi-infarct disease remained confused, both clinically and pathologically. ${ }^{3}$ Nissl also characterised the vascular pathology of Binswanger's disease and much later Bennett et $a l^{4}$ attempted to standardise the antemortem diagnosis. Necessary features were: dementia, bilateral radiological abnormalities (CT or MRI), and two of the following: vascular risk factors; focal cerebrovascular disease; "subcortical" cerebral dysfunction.

J M S PEARCE 304 Beverley Road, Anlaby, East Yorks HU10 $7 B G, U K$

1 Binswanger O. Die Abgrenzung der allgemeinen progressiven Paralyse, I-III. Berl Klin Wochenschr 1894;49:1103-5, 1137-9, 1180-6. (Translation by H Förstl et al. Binswanger on Binswanger's disease and presentation of 2 cases. Intern $\mathcal{f}$ Geriatr Psychiat 1991;6:529-35.)

2 Alzheimer A. Die seelenstorungen auf arteriosclerotischer grundlage. Allgemeine Zeitschrift fur Psychiatrie 1902;59: 695-9.

3 Olszewski J. Subcortical arteriosclerotic encephalopathy: review of the literature of so called Binswanger's disease and presentation of two cases. World Neurology 1962:3.35975 .

4 Bennett DA, Wilson RS, Gilley DW, Fox JH. Clinical diagnosis of Binswanger's disease. F Neurol Neurosurg Psychiatry 1990;53:961-5. 